\title{
To evaluate and compare the Upper lip bite test with the Modified Mallampatti Classification in the prediction of difficult mask ventilation and direct laryngoscopic intubation.
}

\author{
Dr Surmila Khoirom ${ }^{1}$,Dr R.W.Naphade ${ }^{2}$ \\ ${ }^{\text {I}}$ (Department of Anaesthesia, Bharati Vidyapeeth Medical College,,Bharati Vidyapeeth Deemed University, \\ Pune, India) \\ ${ }_{2}^{2}$ (Department of Anaesthesia, Bharati Vidyapeeth Medical College,Bharati Vidyapeeth Deemed University,
} Pune, India)

\begin{abstract}
Background: To find out a better method for airway evaluation. Aims: The aim of the study was to compare the ULBT with Modified Mallampatti Classification in the prediction of difficult mask ventilation and direct laryngoscopic intubation. Settings and design: A prospective double blind, randomized controlled study was conducted in a tertiary centre. Methods: A total of 180 patients were selected for our study. All the patients were assessed using both the Upper Lip bite test and Modified Mallampatti Classification preoperatively by an independent observer. Later the observations of the two tests were compared with the mask ventilation, Cormacke Lehane grade of laryngoscopic view along with ease of intubation by a separate anaesthesiologist . Statistical analysis: Statistical analysis was done using statistics package. P-value less than 0.05 was considered statistically significant. Results:The maximum number of cases were seen in the age group of 19-29. Correlation of both MPC and ULBT was good with obesity. Both MPC and ULBT showed a good correlation with the space available for intubation. However, ULBT was more specific as well as sensitive than MPC in difficult mask ventilation. Conclusion: ULBT is a better bedside test to predict difficult intubation and difficult mask ventilation than MPC.
\end{abstract}

Keywords: Airway management, Difficult laryngoscopy, Intubation, Mask ventilation, Upper lip bite test

\section{Introduction}

Airway is a single priority which always takes precedence, whether it is in the operation theatre, labour suite, casualty department, critical care unit or any scene of resuscitation ${ }^{1}$. Difficult airway, often unexpected, has been identified as the commonest contributory factor to anaesthetic related maternal death ${ }^{2}$. Expertise in airway management is important and is a life line for the anaesthesiologist. It is the responsibility of the anaesthesiologist to secure the airway of the patient. Respiratory compromise accounts for the single largest class of adverse outcomes in the American Society of Anaesthesiologist's Closed Claim Project ${ }^{3}$. According to Robert Caplan ${ }^{4}$, the three commonest causes of respiratory related injuries are inadequate ventilation, oesophageal intubation and difficult intubation. Assessment of the airway becomes a very important step prior to the induction of anaesthesia. Inter-incisor distance $:$ It is the distance between the upper and lower incisors.Normal is $4.6 \mathrm{cmor}$ more, while $<3.8 \mathrm{~cm}$ predicts difficult airway. Modified Mallampatti Classification ${ }^{5}$ has continued to be the single most simple and most commonly employed bedside test to predict difficult intubation till recently. The Upper Lip Bite Test described by Dr.Zahid Hussain Khan ${ }^{6}$ in 2003 has since then replaced the Modified Mallampatti score for predicting difficult intubations. Wilson and colleagues ${ }^{7}$ developed another scoring system in which they took 5 variables- weight, head, neck and jaw movements, mandibular recession,presence or absence of buck teeth.It cannot be used in emergency situations because it was complex, requiring more time. Hence, there is a need to find out a reliable, simple single test of airway with a high index of sensitivity and specificity.

\section{Materials And Methods}

180 patients(out of which 72 males, 108 females), ASA physical status I,II,III requiring general anesthesia and tracheal intubation for elective and semi elective surgeries were included in the study. Patients with ASA grade IV requiring rapid sequence induction, unable to obey verbal commands, mouth opening less than 2.5 fingers were excluded. A written informed consent and institutional ethics committee approval were obtained. Preoperatively all the patients were assessed using both the upper lip bite test and the Modified Mallampatti Classification by an independent observer. Later, the observations of the two tests were compared with the Cormack Lehane grade of laryngoscopic view along with ease of intubation by a separate anaesthesiologist who was unaware of the preoperative results of the aforementioned tests. The number of 
attempts required and any other assistance required eg. external laryngeal manipulation , J shaped stylet, use of Gum elastic troupe were noted. The record of the tests and observations were latter compared post operatively.

Difficult laryngoscopy was defined as not being able to visualize any portion of the vocal cords with the conventional laryngoscope. Difficult intubation was defined as proper insertion of tracheal tube with conventional laryngoscope requiring more than 3 attempts or more than 10 minutes.

After premedication and standard monitoring, anesthesia was induced by either Injection sodium pentothal $(5 \mathrm{mg} / \mathrm{kg})$ or Injection Propofol $(2 \mathrm{mg} / \mathrm{kg})$ as the inducing agents and after confirming the ability to ventilate, Injection Scoline $(2 \mathrm{mg} / \mathrm{kg})$ was used for muscle relaxation.

Ventilation was done with intermittent positive pressure and ease of ventilation was noted and graded. When fasciculation passed from the upper extremity, laryngoscopy was attempted with the head supported by head ring of $10 \mathrm{~cm}$ height, thus achieving flexion at the neck and extension at the atlanto-occipital joint. Standard Macintosh blades- size 3 for females and small males and size 4 for large males was used for laryngoscopy which was done by an experienced anaesthesiologist. Laryngoscopic view was graded as per Cormack Lehane's grades.

Easy intubation was defined as Cormack grade I and II whereas difficult intubation as Grade III and IV on direct laryngoscopic visualization.

Intubation was done with appropriate size PVC endotracheal tube and following points were noted like difficulty in introduction of laryngoscope, space available for intubation, attempts required, aid required for intubation(Bougie/ ILMA/McCoy's/ Fibreoptic bronchoscope).

The findings were documented by an experienced anaesthesiologist and the results were then subjected to statistical calculations. All the results were tabulated and special observations recorded, tabulated and analyzed statistically.

\section{Observations And Results}

180 patients were enrolled, over a period of 1 year. Out of these, 72 were males and 108 were females. Regarding the age and sex wise distribution, the maximum number of cases were seen in the age group of 19-29 years with 75(41.67\%) of which 21(11.67\%) were males and 54(30\%) were females.

Occurrence of anticipated simple intubation(Class I and II) in the normal weight category was $96.43 \%$ and the incidence of anticipated difficult intubation ( Class III and IV) by Modified Mallampatti Classification(MPC) was seen more in obese group with $50 \%$ positive finding. Hence, correlation of MPC is good with obesity.

The findings of anticipated difficult intubations(Class II and III) in all weight categories were $7.89 \%$ in underweight, $8.03 \%$ in normal weight category, $30 \%$ in pre obese group and $30 \%$ in obese group. Hence, correlation of ULBT is good with obesity.

169 patients who were predicted to have easy intubation by MPC had easy intubation(98.22\%) on Direct Laryngoscopic visualization but out of the patients in whom difficult intubation was predicted by MPC(Class III and IV), 10 cases turned out to be easy intubation. Out of 4 cases found to be dififcult intubation, only 1 case was accurately predicted by MPC.

\section{Figures And Tables}

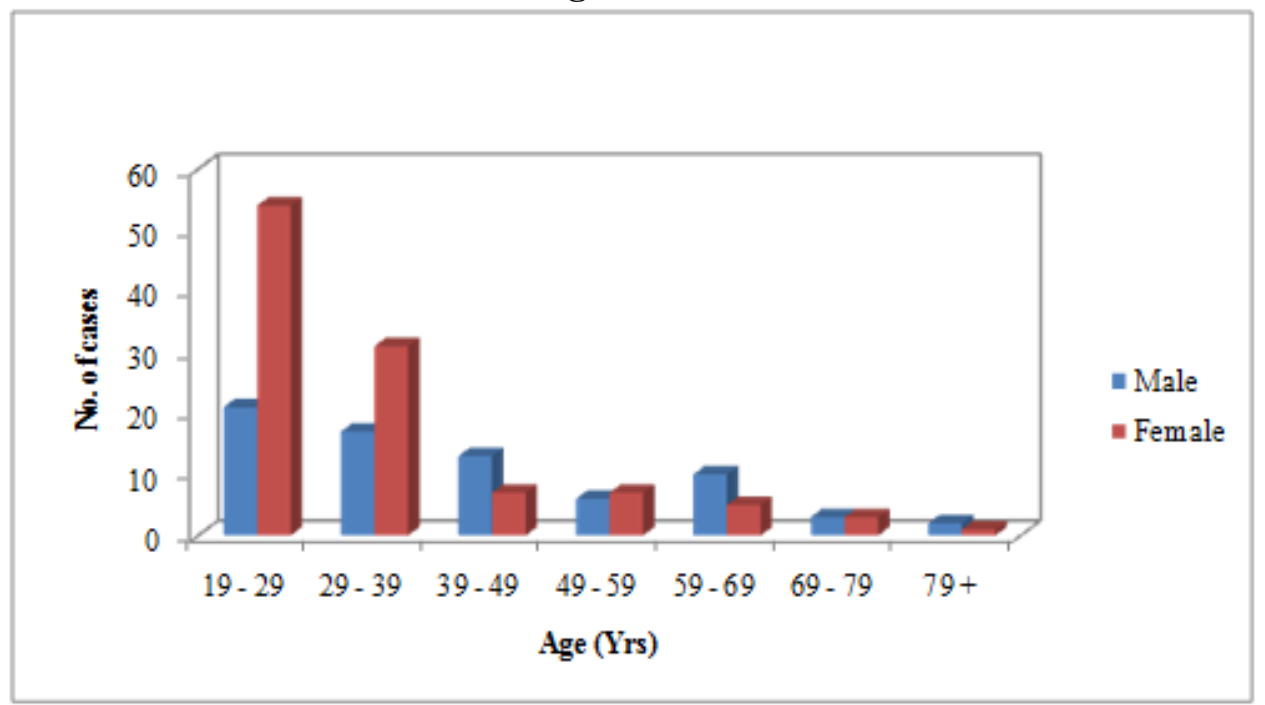

Figure 1: Multiple Bar diagram showing age and sex wise distribution of cases in study group 


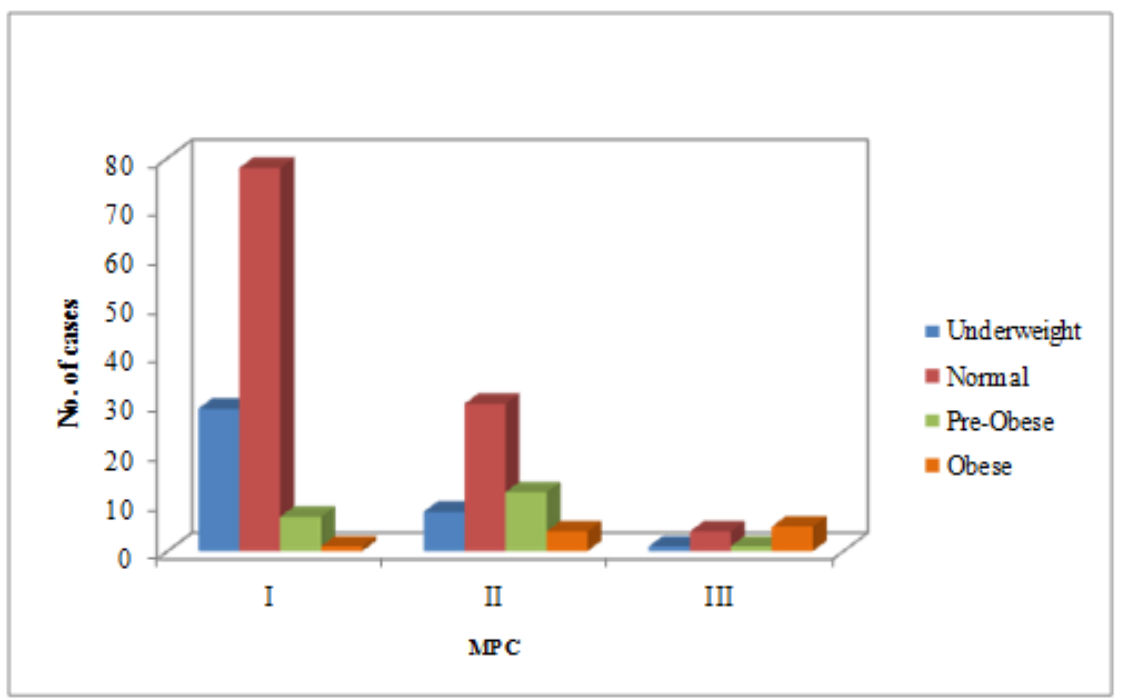

Figure 2: Multiple Bar diagram showing MPC and Body Mass Index in study group

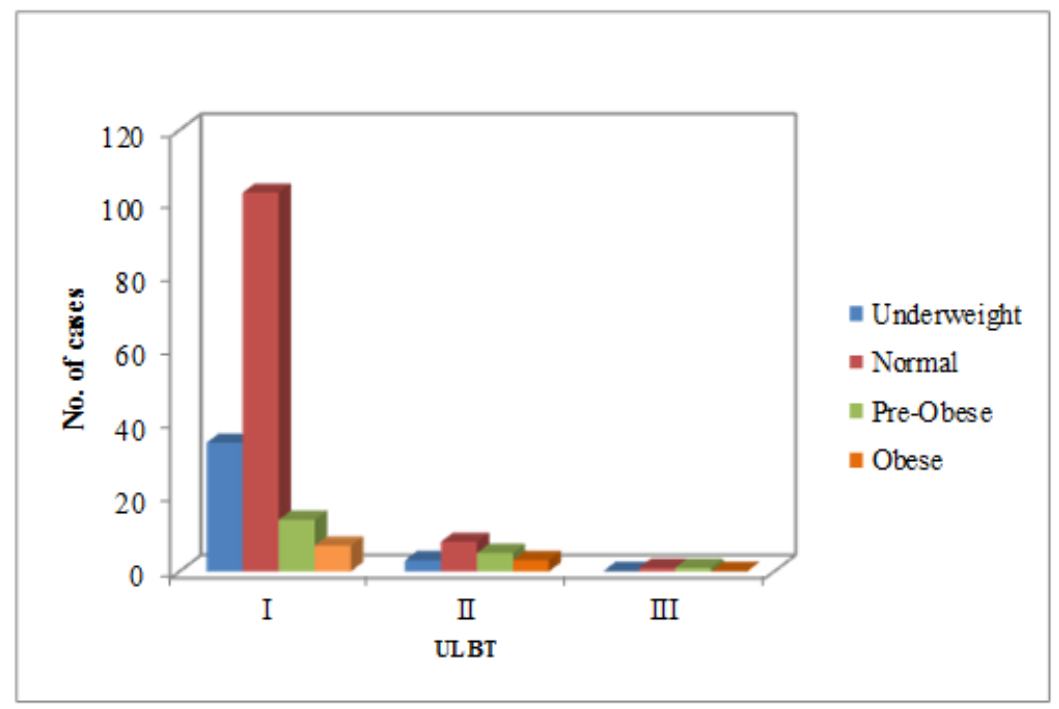

Figure 3: Multiple Bar diagram showing ULBT and Body Mass Index in study group

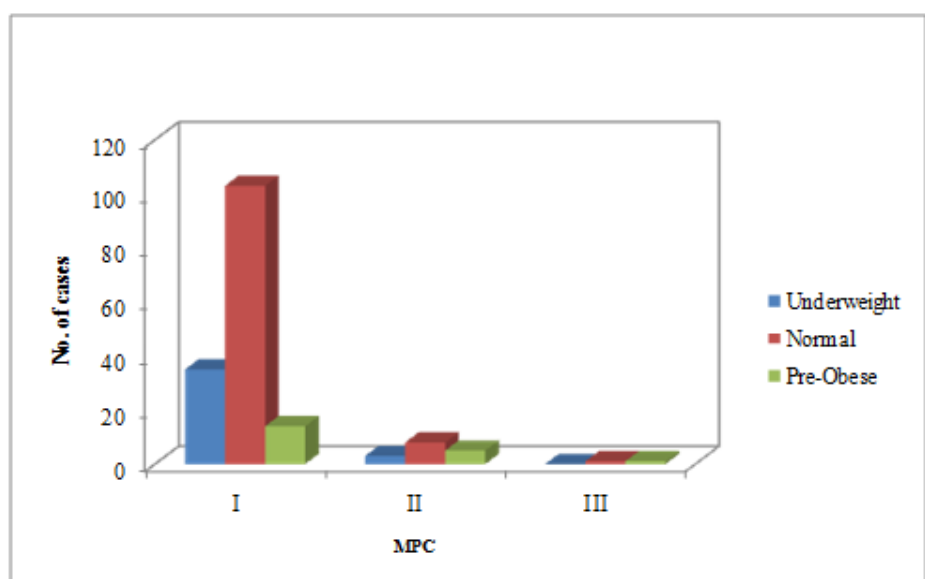

Figure 4: Multiple Bar diagram showing MPC and Cormack Lehane grade in study group 


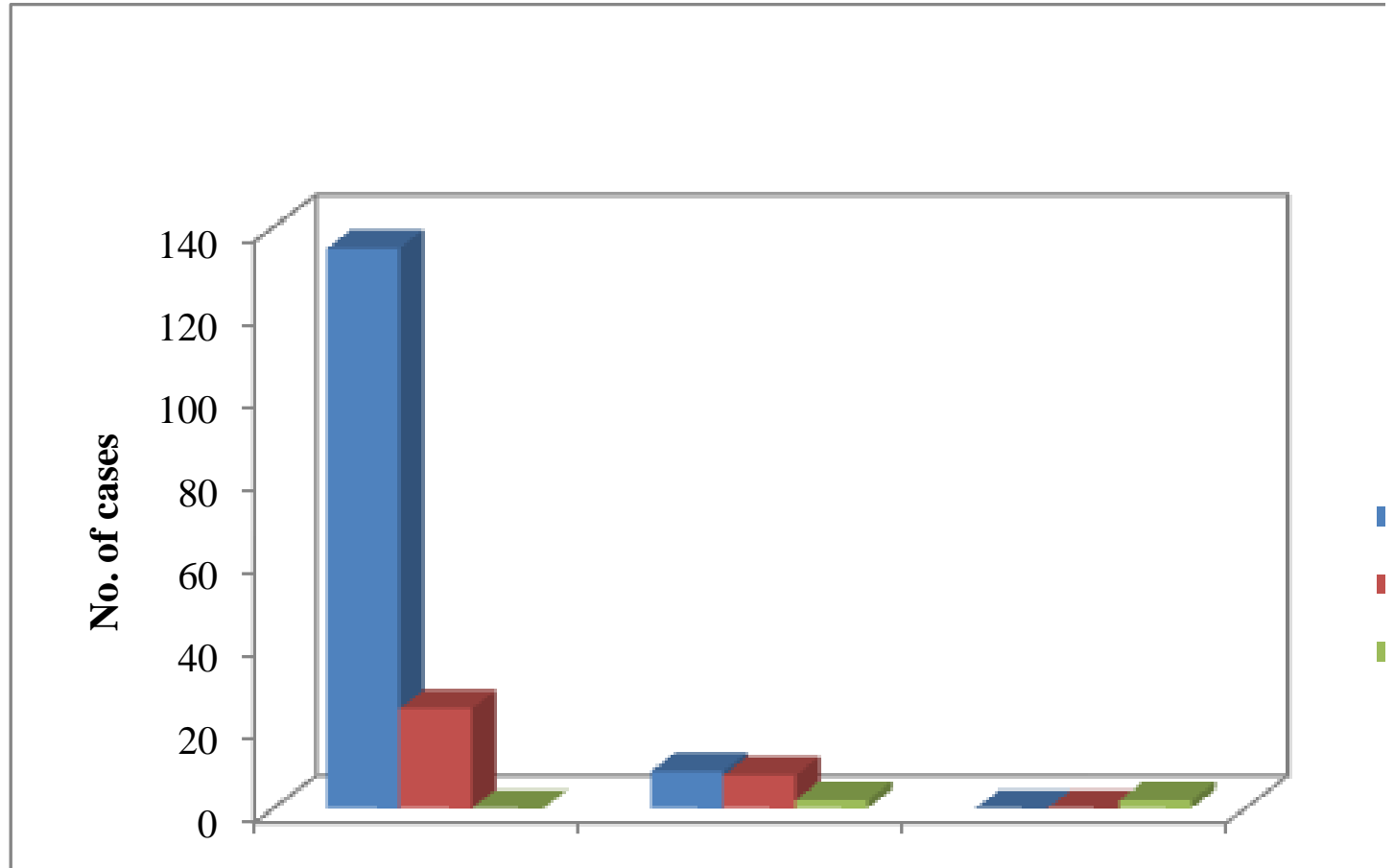

Figure 5: Multiple Bar diagram showing ULBTand Cormack Lehane grade in study group

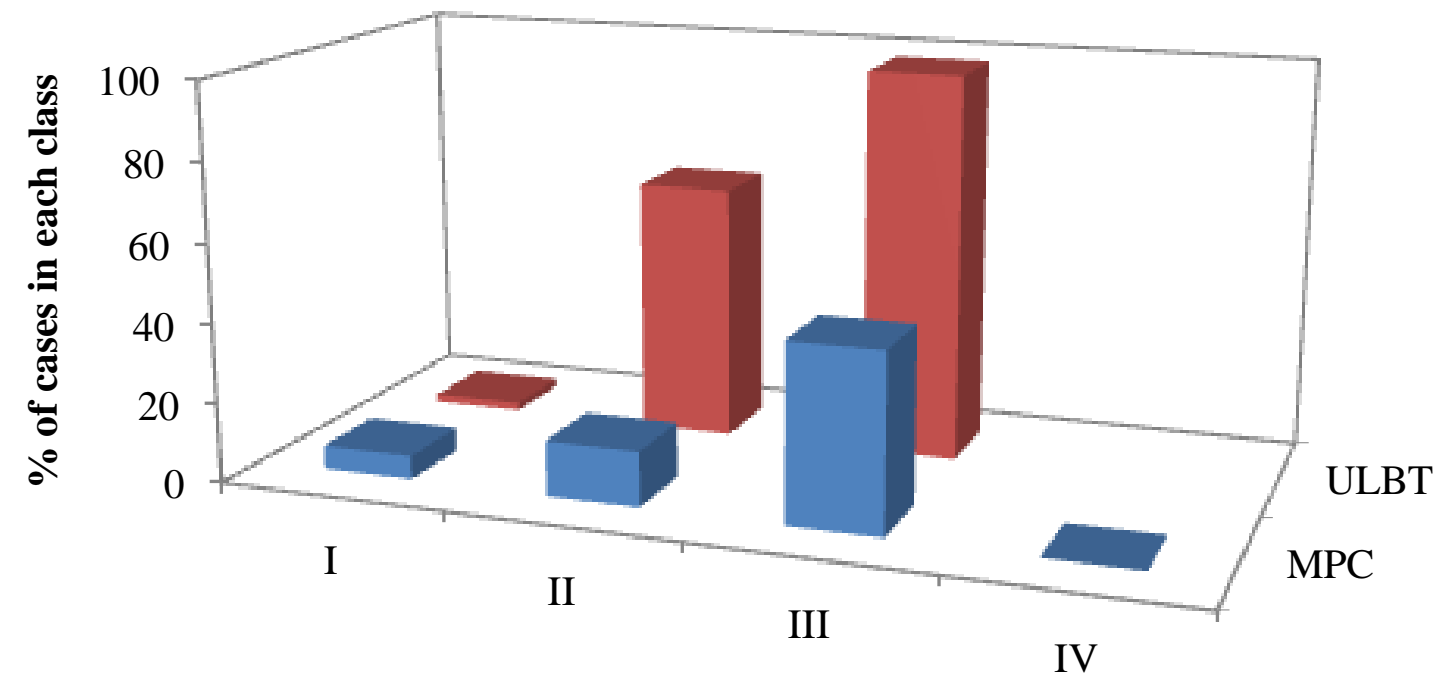

Figure 6: Correlation of MPC and ULBT assisstance required for intubation

TABLE 1: Age and Sex wise distribution of cases in study group

\begin{tabular}{|l|l|l|l|}
\hline \multirow{2}{*}{ Age (Yrs) } & \multicolumn{2}{|l|}{ Sex } & \multirow{2}{*}{ Total (\%) } \\
\cline { 2 - 3 } & Male (\%) & Female (\%) & \\
\hline $\mathbf{1 9}-\mathbf{2 9}$ & $21(11.67)$ & $54(30)$ & $75(41.67)$ \\
\hline $\mathbf{2 9}-\mathbf{3 9}$ & $17(9.44)$ & $31(17.22)$ & $48(26.67)$ \\
\hline $\mathbf{3 9}-\mathbf{4 9}$ & $13(7.22)$ & $7(3.89)$ & $20(11.11)$ \\
\hline $\mathbf{4 9}-\mathbf{5 9}$ & $6(3.33)$ & $7(3.89)$ & $13(7.22)$ \\
\hline $\mathbf{5 9}-\mathbf{6 9}$ & $10(5.56)$ & $5(2.78)$ & $6(8.33)$ \\
\hline $\mathbf{6 9}-\mathbf{7 9}$ & $3(1.67)$ & $3(1.67)$ & $3(1.67)$ \\
\hline $\mathbf{7 9}+$ & $2(1.11)$ & $1(0.56)$ & $\mathbf{1 8 0}(\mathbf{1 0 0})$ \\
\hline Total & $\mathbf{7 2 ( 4 0 )}$ & $\mathbf{1 0 8}(\mathbf{6 0})$ & \\
\hline
\end{tabular}


To evaluate and compare the Upper lip bite test with the Modified Mallampatti Classification in the

TABLE 2: Relationship of MPC and Body Mass Index in study group

\begin{tabular}{|l|l|l|l|l|l|}
\hline \multirow{2}{*}{ MPC } & Body Mass Index & \begin{tabular}{l} 
Tre-Obese \\
Underweight \\
\cline { 2 - 5 }
\end{tabular} & $\begin{array}{l}\text { Normal } \\
(\mathbf{\%})\end{array}$ & $\begin{array}{l}\text { Obese } \\
(\boldsymbol{\%})\end{array}$ & \\
\hline I & $29(16.11)$ & $78(43.33)$ & $7(3.89)$ & $1(0.56)$ & $115(63.89)$ \\
\hline II & $8(4.44)$ & $30(16.67)$ & $12(6.67)$ & $4(2.22)$ & $54(30)$ \\
\hline III & $1(0.56)$ & $4(2.22)$ & $1(0.56)$ & $5(2.78)$ & $11(6.11)$ \\
\hline IV & $0(0)$ & $0(0)$ & $0(0)$ & $0(0)$ & $0(0)$ \\
\hline Total & $38(21.11)$ & $112(62.22)$ & $20(11.11)$ & $10(5.56)$ & $180(100)$ \\
\hline
\end{tabular}

$\mathrm{x}^{2}=49.89, \mathrm{P}<0.0001$

TABLE 3: Relationship of ULBT and Body Mass Index in study group

\begin{tabular}{|c|c|c|c|c|c|}
\hline \multirow[t]{2}{*}{ ULBT } & \multicolumn{4}{|c|}{ Body Mass Index } & \multirow[t]{2}{*}{ Total $(\%)$} \\
\hline & $\begin{array}{l}\text { Underweight } \\
(\%)\end{array}$ & $\begin{array}{l}\text { Normal } \\
(\%)\end{array}$ & $\begin{array}{l}\text { Pre-Obese } \\
(\%)\end{array}$ & $\begin{array}{l}\text { Obese } \\
(\%)\end{array}$ & \\
\hline $\mathbf{I}$ & $35(19.44)$ & $103(57.22)$ & $14(7.78)$ & $7(3.89)$ & 159 (88.33) \\
\hline II & $3(1.67)$ & 8 (4.44) & $5(2.78)$ & $3(1.67)$ & $19(10.56)$ \\
\hline III & $0(0)$ & $1(0.56)$ & $1(0.56)$ & $0(0)$ & $2(1.11)$ \\
\hline Total & $38(21.11)$ & $112(62.22)$ & $20(11.11)$ & $10(5.56)$ & $180(100)$ \\
\hline
\end{tabular}

$\mathrm{x}^{2}=13.69, \mathrm{P}<0.05$

TABLE 4: Relationship of MPC and Cormack Lehane grade in study group

\begin{tabular}{|l|l|l|l|l|}
\hline \multirow{2}{*}{ MPC } & \multicolumn{3}{|l|}{ Cormack Lehane grade } & Total (\%) \\
\cline { 2 - 4 } & I (\%) & II (\%) & & \\
\hline I & $95(52.78)$ & $19(10.56)$ & $1(0.56)$ & $115(63.89)$ \\
\hline II & $42(23.33)$ & $10(5.56)$ & $2(1.11)$ & $54(30)$ \\
\hline III & $7(3.89)$ & $3(1.67)$ & $1(0.56)$ & $11(6.11)$ \\
\hline Total & $144(80)$ & $32(17.78)$ & $4(2.22)$ & $180(100)$ \\
\hline
\end{tabular}

$\mathrm{x}^{2}=4.99, \mathrm{P}<0.05$

TABLE 5: Relationship of ULBT and Cormack Lehane grade in study group

\begin{tabular}{|l|l|l|l|l|}
\hline \multirow{2}{*}{ ULBT } & \multicolumn{2}{|l|}{ Cormack Lehane grade } & Total (\%) \\
\cline { 2 - 4 } & I $(\%)$ & II $(\%)$ & III $(\%)$ & \\
\hline I & $135(75)$ & $24(13.33)$ & $0(0)$ & $159(88.33)$ \\
\hline II & $9(5)$ & $8(4.44)$ & $2(1.11)$ & $19(10.56)$ \\
\hline III & $0(0)$ & $0(0)$ & $2(1.11)$ & $2(1.11)$ \\
\hline Total & $144(80)$ & $32(17.78)$ & $4(2.22)$ & $180(100)$ \\
\hline
\end{tabular}

$\mathrm{x}^{2}=107.41, \mathrm{P}<0.0001$

TABLE 6: Relation of assistance required for intubation in relation to both the tests

\begin{tabular}{|l|l|l|l|l|l|}
\hline Tests & Help taken & Class I & Class II & Class III & Class IV \\
\hline \multirow{2}{*}{ MPC } & J shaped & 4 & 3 & 1 & 0 \\
\cline { 2 - 6 } & Others & 2 & 3 & 4 & 0 \\
\hline \multirow{2}{*}{ ULBT } & J shaped & 1 & 6 & 1 & \\
\cline { 2 - 6 } & Others & 2 & 6 & 1 & \\
\hline
\end{tabular}

\section{Discussion}

In this study, we had included 180 patients in which both MPC and ULBT was assessed preoperatively. Later documentation was made regarding ease of mask ventilation, ease of laryngoscope insertion, grade of laryngoscopic view, space available for intubation, attempts required for intubation and assistance required for accomplishment of intubation in the form of ' $\mathrm{J}$ ' shaped stylet and external laryngeal manipulation (BURP maneuver). We attempt to correlate all these findings with MPC and ULBT. While mask ventilating the patients ,it was found that out of total 180 patients,162(90\%)could be ventilated by one hand,15(8.3\%)required two hands and 3(1.6\%)required airways (oropharyngeal/nasopharyngeal or both). These observations were compared with preoperative MPC grades. Out of 115 patients belonging to MPC Class I, 115(100\%)patients could be ventilated with only one hand. None of the above patients required both hands and airway adjuvants for ventilation. Out of 54 patients of MPC Class II, 40(74.07\%)required only one hand ,12(22.22\%)required two hands and 2(3.70\%)required airways. In MPC Class III, out of 11patients, 7(63.64\%) required only one hand for ventilation and $3(27.27 \%)$ required two hands and only $1(9.09 \%)$ required help of airways for mask ventilation. The above observations were compared with preoperative ULBT grades. Out of 159 patients belonging to ULBT Class I,152(95.59\%)patients could be ventilated with only one hand, 5(3.14\%)required two hands for 
successful ventilation and 2(1.25\%) of all the patients in Class I required airway adjuvants. Out of 19 patients of ULBT Class II, 10(52.63\%)required only one hand, 8(42.11\%) required two hands and 1 (5.26\%)required airways. In ULBT Class III which had 2 patients, both required two hands for mask ventilation. Sensitivity of MPC and ULBT for requirements of two hands and airway adjuvants to maintain mask ventilation were $22.22 \%$ and $61.11 \%$ respectively, whereas, specificity is almost similar i.e. $95.68 \%$ and $93.83 \%$ respectively. Positive predictive value of MPC was $36.36 \%$ and ULBT was 52,38\% ; negative predictive value was $91.72 \%$ and 95.59\% respectively. 166 patients had sufficient space for intubation and 14 patients had just enough space for intubation. Out of these 14 patient $(42.86 \%)$ belonged to MPC Class I ,3(21.43\%)belonged to Class II and $5(35.71 \%)$ belonged to Class III. Out of 14 patients having just enoughspace for intubation ,6(42.86\%)belonged to ULBT Class I; 7(50\%)belonged to Class II and I(7.14\%)belonged to ClassIII of ULBT.]'।

Sensitivity of MPC and ULBT for availability of space for intubation were $35.71 \%$ and $57.14 \%$ whereas specificity was almost the same that is $96.39 \%$ and $92.17 \%$.Positive predictive value of MPC was $45.45 \%$ and ULBT was $38.10 \%$; negative predictive values were $94.67 \%$ and $96.23 \%$.

In our study, 17 patients required assistance for intubation; 6(5.22\%)belonged to MPC Class I, 6(11.11\%)to Class II and 5(45.45\%)to Class III. When seen in reference to ULBT,3 (1.8 9\%) of Class I, 12(63.14\%)of Class II and 2(100\%)of Class III required assisted intubation.

The sensitivity of MPC was $25 \%$, specificity was $94.32 \%$,positive predictive value was $9.09 \%$ and negative . predictive value was $98.22 \%$ and for ULBT sensitivity was $100 \%$ specificity was $90.34 \%$,positive predictive value was $19.05 \%$ and negative predictive value was $100 \%$.

At last,we confirmed that the sensitivity, negative predictive value as well as positive predictive value of ULBT is more than the MPC. The specificity and accuracy of both the tests were nearly the same. So, overall it can be concluded that, ULBT could predict nearly all cases of difficult intubations; it is a better single bedside test of airway assessment. ULBT predicts difficult mask ventilation with sensitivity of over $61.11 \%$ vs MPC with a sensitivity of $35.71 \%$. Hence, we conclude that ULBT is a better test than MPC for predicting difficult and mask ventilation.

[1] Editorial-Indian Journal of Anaesthesia 2005;49(4):248-250.

[2] Rocke DA, Murray WB, Rout CC. Relative risk analysis of factors associated with difficult intubation in obstetric anaesthesia. Anaesthesiology 1992:77:67-73.

[3] Randell T. Prediction of difficult intubation. Acta Anesthesio Scand 1996;40:1016-23.

[4] Robert Caplan. Practical guidelines for management of the difficult airway, a report by ASA task force on management of difficult airway. Anaesthesiology 1993, 78:597-692.

[5] Editorial - Indian Journal of Anaesthesia. 2005;49(4):258-262.

[6] Khan ZH, Kashfi A, Ebrahimkhani E. A comparison of upper lip bite test(a simple new technique) with modified Mallampatti Classification in predicting difficult endotracheal intubation: a prospective blind study. Anesth Analg 2003; 96:595-599.

[7] Wilson ME, Spiegelhalter D, Robertson JA, Lesers P. Predicting difficult intubation. British Journal of Anaesthesia 1988; 61:211216. 